\title{
Sensitive skin: a complex and multifactorial syndrome
}

\author{
A Pons-Guiraud \\ Department of Dermatology, Hôpital Saint Louis, Paris, France
}

\begin{abstract}
Summary
Many people complain of discomfort after application of commonly used skin care products, particularly to the face. This hyperreactivity of the skin is a non-immunologically mediated skin inflammation. It seems to be the result of an intolerance of the skin to various stimuli that are normally well tolerated. It is difficult to assess the prevalence of 'sensitive skin' and sensitive skin-related cosmetic intolerance because of the many possible exogenous and endogenous factors that trigger or aggravate this multifactorial syndrome.

A thorough history is essential. Sometimes patch testing is needed both to standard allergens and also to all the patients' cosmetics and skin care products. All cosmetics should be stopped and then reintroduced one by one, at intervals of one to two weeks. The number, type and frequency of the application of skin care products used in the final programme should remain limited. Some patients benefit from psychological or even psychiatric help. Management is usually difficult and all therapeutic measures should be undertaken with patience and tenacity.
\end{abstract}

Keywords: cosmetic intolerance, reactive skin, sensitive skin, stinging test

\section{Introduction}

During the last few years there has been a growing number of patients with a subjective sensitive skin discomfort. They complain of particular susceptibility when applying commonly used skin care products i.e. cosmetics, soaps and sunscreens, without classical visible signs of irritation, contact allergy, phototoxicity or photocontact.

Essentially a self-diagnosed condition, this syndrome is later aggravated by environmental factors and phenotypic or genotypic features. Clinically subjective symptoms can be very intense, objective signs are very poor or absent.

To confirm a diagnosis of sensitive skin, a detailed personal and family history and physical examination are necessary. From time to time, patch and photopatch

Correspondence: Annick Pons-Guiraud, Department of

Dermatology, Hôspital Saint Louis, Paris, France,

E-mail: annick.pons-guiraud@wanadoo.fr

Accepted for publication 12 August 2004 tests are needed to rule out mild or subclinical manifestations of contact allergy. In these cases, patch testing can be done using screening series and also all of the patient's cosmetics and skin care products.

Management of sensitive skin is always difficult for both the physician and the patient. It is necessary to find a regimen of facial skin care that does not cause discomfort.

\section{Epidemiology}

Sensitive (or hyperirritable) skin is a common problem among women and men and these consumers of skin care products complain of a reduced tolerance to frequent or prolonged use of cosmetics. ${ }^{1}$

Willis, et al. ${ }^{2}$ carried out an epidemiological study in the UK. They performed a random study by questionnaires sent out to 3300 women and 500 men to assess the prevalence of sensitive skin cosmetic-related sideeffects and to examine the possible factors that may be associated. The response rates were $62 \%$ for women and $52 \%$ for men with the incidence of self-reported skin 
sensitivity being, respectively, $51.4 \%$ and $38.2 \%$. Ten per cent of women and $5.8 \%$ of men described themselves as having very sensitive skin. Fifty-seven per cent of women and $5.8 \%$ of men had experienced an adverse reaction to a product at some stage in their lives, with $23 \%$ of women and $13.8 \%$ of men having had a problem in the last 12 months. Among the women, symptoms of cosmeticinduced subjective sensory skin discomfort including burning, stinging, itching, etc., occurred more commonly in the sensitive skin cohort (53\%) than in those who regarded themselves as nonsensitive (17\%).

P.J. Frosch and A.M. Kligman ${ }^{3}$ investigated skin responses to various irritants, and showed that 14\% of sensitive skins in a normal population is related to a thin, permeable stratum corneum barrier.

According to Adams and Maibach, ${ }^{4}$ most are female $(79 \%)$ and $85 \%$ are White people. Racial differences in cutaneous irritability have been well documented. Weigand ${ }^{5}$ has shown that the stratum corneum of Black people has more cell layers on average than that of White people. This group also found that the buoyant density of black stratum corneum was higher, which may indicate a more compact barrier.

In another study, this view is challenged: the measure of the transepidermal water loss (TEWL) shows a higher susceptibility of the skin in Black people compared to White people. $^{6}$

\section{Clinical presentation}

Essentially a self-diagnosed condition, this syndrome is later aggravated by environmental factors and phenotypic or genotypic features.

\section{Symptoms}

Patients bitterly complain of subjective symptoms such as burning, stinging, prickling, itching or tight-feeling. It's always difficult to quantify the intensity and the nature of these symptoms which can vary sharply from one individual to another. Some do not tolerate any skin care and cosmetic products, even simple formulations, applied on the face and much more rarely on the scalp and the neck.

This discomfort worsens after each application of any cosmetic product; the onset is usually rapid, within minutes, but patients also notice symptoms hours later. They always apply new cosmetics but after the persistence of this subjective irritation, they usually eliminate all cosmetics. This skin discomfort persists for either a more or less period of time before becoming permanent, obsessional and unbearable. Fisher ${ }^{7}$ coined the term 'status cosmeticus' for this extreme intolerance to cosmetics.

\section{Signs}

Objective signs are very poor or absent. The physicians sometimes observe skin dryness, facial erythema, erythrocoupe rose or fine scaling. In fact, they often have to cope with an 'invisible dermatosis', ${ }^{8}$ showing no sign of inflammation or irritation. This hyperreactivity of the skin is more frequently observed in women with dry and light skin complexions (phenotype II). But men with thick, fatty or dry skin may also have sensitive skin, and complain of intolerance to all cosmetics, shaving products and moisturizing creams.

In all cases, sensitive skin is a multifactorial syndrome.

In numerous people this hyperreactivity is genetic but many factors can trigger or aggravate it.

\section{Exogenous features}

The use of soap and hygiene products of cosmetics, sunscreens, chemical exfoliants not suited to the patient's skin type. Some chemicals such as alcohol, propylene glycol, butylene glycol, cocamidopropylbetaine, triethanolamine are common offending subjective irritants which can be found in many formulations. ${ }^{9}$ Some peelings with exfoliants i.e. resorcine, TCA, AHA, depending on the concentration, on the $\mathrm{pH}$ in the final formulation are often very irritating and an aggravating factor on hyperirritable skin. They often induce an inflammatory response after repeated exposures at the same fragile site such as eyelids and lead to a cumulative irritation, remaining invisible for a long time. Furthermore, local trauma sunburn, phototherapy or UVA exposures, or a cosmetic procedure such as dermabrasion, laser resurfacing or facelift can aggravate this syndrome. A surgery of the face disturbs the self image and on a depressed or neurotic background, may trigger an acute and permanent feeling of discomfort.

Some other environmental factors induce a light irritation: cold, sun, wind, heat, pollution. Irritating diets containing spices, alcohol, coffee and the ingestion of very hot beverages trigger bouts of flushing for a more or less short time.

Furthermore, daily topical corticotherapy, very often prescribed by physicians for an 'allergic' pathology, makes the skin increasingly fragile, subject to more and more intense erythema which quickly becomes permanent and intolerant to all cosmetic products, resulting in a 'steroid dermatitis'.

\section{Endogenous features}

Among the patients complaining of having sensitive skin, endogenous dermatoses can be atypical and remain unknown. They are often masked by a corticosteroid 
local therapy or aggravated by an ill-suited topical treatment. Thus, they often make the skin very fragile and induce an irritative reaction to skin care products and cosmetics.

Hence, pre-existing skin conditions i.e. seborrheic diathesis, atypical psoriasis, rosacea and perioral dermatitis, an erythro-couperosis with bouts of flushing or essentially a residual atopic dermatitis must be investigated and identified. However, for some investigators, an atopic diathesis in women did not appear to be a predictive factor for sensitive skin, the incidence of self-perceived sensitive skin being equivalent for atopics (49\%) and nonatopics (51\%) and some $34 \%$ of atopic women described themselves as being nonsensitive. Nevertheless, in the same study, the investigations point out that the incidence of atopy was higher among women in the sensitive skin group (49\%) than among those in the nonsensitive group $(27 \%)^{2}$

Furthermore, some people have a disturbed image of their body or face which can lead to complaints of physical defects and feelings without any objective signs.

In fact, a temporary or permanent conjunction of quite a few of these exogenous and endogenous factors is responsible for this hyperreactivity of the skin.

\section{Clinical forms}

Depending on the intensity of the clinical symptoms, several clinical forms have been described: ${ }^{2}$

- 'very sensitive skin' dry or fatty, bitterly reacting both to exogenous factors, i.e. cosmetic products and environmental factors and endogenous features. The clinical symptoms are acute and permanent, and both triggered determining psychological reactions;

- 'environmentally sensitive skin', often clear, dry and thin skin, essentially reactive to environmental factors i.e. heat and rapid temperature changes, with frequent bouts of flushing; and

- 'cosmetically sensitive skin', essentially reactive to cosmetics. This intolerance is lighter and often limited to some identifiable cosmetic products.

\section{Diagnosis}

Before finalizing the diagnosis of sensitive skin, it is necessary to establish a comprehensive questionnaire completed by a physical examination, a detailed personal and family history and, from time to time, patch and photopatch tests to rule out mild or subclinical manifestations of contact allergy. In these cases, patch testing will be made with a series of screening and with all the patient's cosmetics and skin care products. Sometimes it will be interesting to repeat the application for 14 days, twice a day (ROAT) on the antecubital fossa to eliminate mild irritation or an allergy. ${ }^{10}$ For some investigators, the patch test to nickel sulphate appears statistically significant between sensitive and nonsensitive skin. In most nickel sensitive patients, after the interruption of the content allergens and the use of better quality of cosmetic products, they observe an improvement of the dermatitis. $^{11}$

With a view to finding an objective criterion, Frosch and Kligman ${ }^{3}$ have carried out a test that compares a single application on the nasolabial fold of $0.05 \mathrm{~mL}$ of a $10 \%$ lactic acid solution, to the distilled water applied on the contralateral site at room temperature. Depending on the hyperreactivity of the skin this test triggers a more or less stinging reaction classified on an arbitrary scale in four classes. Issachar et al. studied the $\mathrm{pH}$ measurement during the lactic acid test on nasolabial fold. On sensitive skin, they observed an increase of $\mathrm{pH}$ faster than in normal skin. Thus, this test can confirm this type of skin and could be a predictive cosmetic-related case of adverse reactions.

Recently, an application of capsaicin has been proposed to diagnose sensitive skin, thanks to the important cutaneous discomfort of these types of skin compared to the nonsensitive skin. This test could also be predictive for the diagnosis of sensitive skin. ${ }^{12}$

\section{Pathophysiology}

Sensitive skin involves an orthoergic and not an immunological mechanism. ${ }^{13}$ It is an inflammatory reaction resulting from the abnormal penetration in the skin of potentially irritating substances because of the alteration of the barrier function. This barrier is emphasized as being a major feature in this skin discomfort, depending on the thickness of the stratum corneum and the quality of the intracellular lipids. Ceramids are probably the main element in the storage of water in the stratum corneum. Hence, in some disorders i.e. atopic dermatitis and seborrheic dermatitis, the modification of intercorneocytic lipids induces a decrease in the skin tolerance threshold and thus makes it more sensitive to irritants and exogenous stimuli. Even a mild and unsuspected irritation leads to inflammatory responses with a release of arachidonic acid metabolites with increase of E2 and F2 prostaglandines and leucotrienes. ${ }^{14,15}$

More recently, some authors insisted on the involvement of the cutaneous sensory innervation, highlighted by the triggering of sharp stinging after the application of lactic acid. ${ }^{16}$ 
Table 1 Management of sensitive skin.

Apply the smallest possible number of cosmetic products. Choose those intended for 'sensitive skin'.

Choose fragrance-free formulations.

Avoid soaps.

Use non-rinsing cleansing lotions or thermal spring water spritzers.

Do not forget to thoroughly dry the area by gently patting with a paper tissue (do not use cotton wool).

Choose moisturizing creams with a mild texture or even cold creams or cerates.

In case of exposure to air conditioning or to overly heated environments, do not hesitate to reapply these creams several times per day.

Choose hair products without irritating tensio-active surfactants.

Avoid skin cleansing and exfoliating masks.

Avoid applying products containing AHA, retinaldehyde or tretinoin.

If any cosmetic product application is responsible for burning and discomfort, discontinue use immediately.

Protect skin from temperature changes, sunlight, wind and exposure to heat.

Consumption of alcohol must be limited as much as possible.

Observe whether the skin is more irritable after intake of coffee or spices.

If necessary, treat depression and neuropsychiatric signs.

After 3-6 months avoidance of skin care products, progressively reintroduce cosmetic products one by one and at intervals of one or two weeks. Remember that a recurrence is always possible.

\section{Treatment}

The care and management of sensitive skin is always difficult for the physician and the patient. It is mandatory to find a regimen of facial skin care that does not cause discomfort. Recommendations include many points listed above.

\section{Conclusion}

Despite progress in the understanding of this very common and plurifactorial sensitive skin syndrome, its care remains very challenging and requires cooperation from the patient and tenacity from the physician.

\section{References}

1 Pons-Guiraud A. Tolérance aux cosmétiques. Le point en 1995. Nouv Dermatol 1995; 14: 514-21.

2 Willis CM, Shaw S, de la Charriere O, Baverel M, Reiche K, Jourdain Bastien P, Wilkinson JD. Sensitive skin: an epidemiological study. Br J Dermatol 2001 August; 145 (2): 258-63.

3 Frosch PJ, Kligman AM. Method for appraising the stinging capacity of topically applied substances. J Soc Cosmetic Chemist 1977; 28: 197-209.

4 Adams RM, Maibach HI. A 5-year study of cosmetic reactions. J Am Acad Dermatol 1985; 13: 1062-9.

5 Weigand DA, Haygood C, Gaylor JR. Cell layers and density of negro and Caucasian stratum corneum. J Invest Dermatol 1974; 62: 563-8.

6 Berardesca E, Maibach HI. Racial differences in sodium lauryl sulphate induced cutaneous irritation: black and white. Contact Dermatitis 1998; 18: 65-70.

7 Fischer AA. Part I: 'Status cosmeticus'; a cosmetic intolerance syndrome. Cutis 1990; 46: 109-10.

8 Kligman AM. The invisible dermatosis. Arch Dermatol 1991; 127: 1375-82.

9 Basketter DA, Griffiths HA. A study of the relationship between susceptibility to skin stinging and skin irritation. Contact Dermatitis 1991; 29: 185-8.

10 Issachar N, Gall Y, Borelll MT, Poelman MC. pH measurements during lactic acid stinging test in normal and sensitive skin. Contact Dermatitis 1997; 36: 152-5.

11 Francomano M, Bertoni L, Seidenari S. Sensitive skin as a subclinical expression of contact allergy to nickel sulfate. Contact Dermatitis 2000; 42: 169-70.

12 de la Charriere O, Reich L, Montastier C, Nicholson M, Courbiere C, Willis C. Skin reaction to capsaicin: a new way for the understanding of sensitive skin. Proceedings of the 19th World Congress of Dermatology (Sydney, Australia), Australas. J Dermatol 1997; 38 (S2): 288.

13 de la Charriere O, Jourdain R, Bastien P, Garrigue JL. Sensitive skin is not a subclinical expression of contact allergy. Contact Dermatitis 2001; 44: 131-2.

14 Rougier A, Lotte C, Corcuff P, Maibach HI. Relationship between skin permeability and corneocyte size according to anatomic site, age and sex in man. J Soc Cosmet Chem 1988; 39: 15-26.

15 Di Nardo A, Sugino K, Wertz P, Ademola J, Maibach HI. Sodium lauryl sulphate (SLS) induced irritant contact dermatitis. a correlation between ceramids and in vivo parameters of irritation. Contact Dermatitis 1996; 35: 86-91.

16 Hosipovitch G. Evaluating subjective irritation and sensitive skin. Cosmetics Toilettries 1999; 114: 41-2. 